\title{
Commentary: First, get the patient off the table
}

\author{
Riyad Karmy-Jones, MD, FACS, FRCSC, ${ }^{\mathrm{a}}$ and \\ Robert DuBose, MD, FACS ${ }^{b}$
}

Luthra and Tsang $^{1}$ present a cogent argument that performing frozen elephant trunk (FET) procedure concurrently, using hybrid stent devices, with repair of ascending aortic dissection should be the standard if "performed at high volume aortic centers."

There are obviously 2 operative considerations: ascending/arch repair and distal stenting. There are several techniques for both steps, including using the hybrid grafts the authors recommend, deploying endografts distally into the descending aorta by a variety of techniques, and hemiarch or total aortic replacement. ${ }^{2}$ Both total aortic replacement and FET appear to be associated independently with reduced mortality on follow-up, albeit both technically more challenging with potential for increased operative mortality and significant complications. ${ }^{3,4}$

The authors' review of the literature regarding FET highlights a report with an excellent 5-year false lumen thrombosis rate $(72 \%)$ with concurrent 5-year freedom from aortic intervention (86.8\%). Alternatively, Kreibich and colleagues ${ }^{5}$ documented a $64 \%$ incidence of aortic reintervention (for a variety of pathology) at 36 months following FET (including intended completion, anticipated, and unexpected reinterventions). These and others' experiences suggest that reintervention (planned or otherwise) is an acceptable approach in centers of excellence.

What is a reproducible marker of experience and high-volume is ill-defined. For example, does a cardiac and/or vascular group that performs a number of fenestrated abdominal endografts and thoracic endovascular aortic

\footnotetext{
From the ${ }^{\mathrm{a} D i v i s i o n}$ of Thoracic/Vascular Surgery, PeaceHealth Southwest Washington Medical Center, Vancouver, Wash; and ${ }^{\mathrm{b}}$ Division of Cardiothoracic Surgery, Oregon Health and Sciences University, Portland, Ore.

Disclosures: The authors reported no conflicts of interest.

The Journal policy requires editors and reviewers to disclose conflicts of interest and to decline handling or reviewing manuscripts for which they may have a conflict of interest. The editors and reviewers of this article have no conflicts of interest.

Received for publication July 7, 2021; revisions received July 7, 2021; accepted for publication July 7, 2021; available ahead of print July 14, 2021.

Address for reprints: Riyad Karmy-Jones, MD, FACS, FRCSC, Division of Thoracic/Vascular Surgery, PeaceHealth Southwest Washington Medical Center, 505 87th Ave, Vancouver, WA 98664 (E-mail: rkarmy-jones@peacehealth.org). J Thorac Cardiovasc Surg 2023;165:589-90 $0022-5223 / \$ 36.00$

Copyright (c) 2021 by The American Association for Thoracic Surgery https://doi.org/10.1016/j.jtcvs.2021.07.004
}

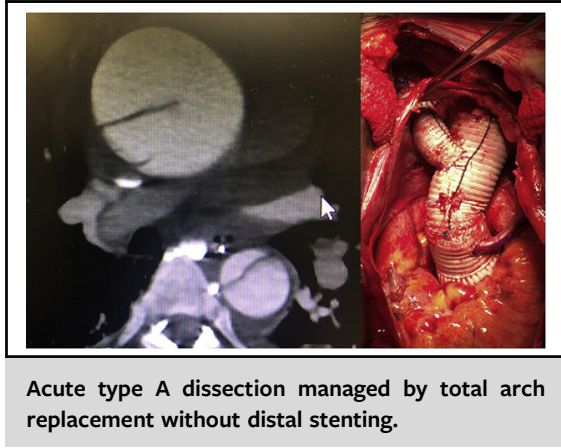

CENTRAL MESSAGE

Distal aortic stabilization after repair of type $A$ dissection results in improved outcomes, but the timing and technique requires continued reassessment by individual centers.

repair for traumatic aortic injury have the "requisite volume of experience" to have the optimal outcome for FET procedures? Would this expertise translate to planned delayed interventions resulting in better, equivalent, or worse outcomes than performing FET at the time of arch repair? Does a cardiac group that manages a lot of emergency type A dissections have the ability to easily transition this to the FET procedure?

Thus, it is possible to assess the authors' central message in portions. They argue that FET has improved outcomes without increased operative risk, and they present compelling arguments. They specifically discuss hybrid stent devices but there are a variety of tools for achieving the same ends. They do not specifically address the difference between hemiarch and total aortic replacement, but comment that in the setting of "limited skills and expertise," "conservative resection" is the answer. The cornerstone of their argument is that these procedures need to be done by "experienced surgeons in high volume aortic centers" and if done so are not associated with increased operative risk but do have better long-term outcomes. I believe their perspective statement has more power than their central message, namely that there needs to be continued multi-institutional coordinated efforts to assess which techniques to use and when to use them. Until then, individual clinical judgment is still required to determine how to achieve primum non nocere, which in this case means, "get the patient off the table alive." 


\section{References}

1. Luthra S, Tsang GM. Concurrent stabilization of 'downstream' aorta during acute type A aortic dissection repair. J Thorac Cardiovasc Surg. 2023;165:586-8.

2. Czerny M, Schmidli J, Adler S, van den Berg JC, Bertoglio L, Carrel T, et al; EACTS/ESC+VS scientific document group. Current options and recommendations for the treatment of thoracic aortic involving the aortic arch: an expert consensus document of the European Association for Cardio-Thoracic surgery (EACTS) and the European Society for vascular Surgery (ESVS). Eur J Cardiothorac Surg. 2019;55:133-62.
3. Matalanis G, Ip S. Total arch repair for acute type A dissection: a new paradigm. J Vis Surg. 2018;4:79-89.

4. Poon SS, Tian DH, Yan T, Harrington D, Nawaytou O, Kuduvalli M, et al. Frozen elephant trunk does not increase incidence of paraplegia in patients with acute type A aortic dissection. J Thorac Cardiovasc Surg. 2020;159: 1189-96.

5. Kreibich M, Berger T, Rylski B, Chen Z, Beyersdorf F, Siepe M, et al. Aortic reinterventions after the frozen elephant trunk procedure. J Thorac Cardiovasc Surg. 2020;159:392-9.e1. 